\title{
REFLECTANCE ANISOTROPY MEASUREMENTS USING A PUSHBROOM SPECTROMETER MOUNTED ON UAV AND A LABORATORY GONIOMETER - PRELIMINARY RESULTS
}

\author{
Juha Suomalainen ${ }^{\mathrm{a}, *}$, Peter Roosjen ${ }^{\text {a }}$, Harm Bartholomeus ${ }^{\mathrm{a}}$, Jan Clevers ${ }^{\mathrm{a}}$ \\ ${ }^{a}$ Laboratory of Geo-Information Science and Remote Sensing, Wageningen University, Netherlands
}

UAV-G 2015, Toronto, Canada

KEY WORDS: UAV, Hyperspectral, Pushbroom, BRF, BRDF, Goniometer

\begin{abstract}
:
During 2014-2015 we have developed a new method to measure reflectance factor anisotropy using a pushbroom spectrometer mounted on a multicopter UAV. In this paper/presentation we describe the acquisition method and show the preliminary results of the experiment. To validate the measurements the same targets have also been measured with a laboratory goniometer system. The first experiments over sugar beet fields in 2014 show similar trends in both UAV and laboratory anisotropy data, but also some differences caused by differences in sampling and diffuse illumination. In 2015 a more extensive study on wheat, barley and potato fields were performed. The measurements were repeated on three days over the growth of the crops allowing linking the development of the crops to the anisotropy signals. On each day the anisotropy measurement was repeated $4-5$ times with different solar zenith angles ranging from $60^{\circ}$ to $40^{\circ}$ allowing analysis how the solar angle affects the anisotropy. The first results of these experiments will be presented in this conference.
\end{abstract}

\section{UAV HARDWARE}

The WageningenUR Hyperspectral Mapping System (HYMSY) (Suomalainen, 2014) is a lightweight camera system developed specifically for UAV based remote sensing (Figure 1). HYMSY consists of a lightweight pushbroom spectrometer $(450-950 \mathrm{~nm}$, FWHM $9 \mathrm{~nm}$ ), a consumer photo camera and a GPS-INS system. Normally HYMSY is mounted under an octocopter UAV to collect hyperspectral maps in traditional pushbroom fashion where the scan line is kept perpendicular to the direction of propagation. However, the flexibility of the octocopter platform allowed us to use HYMSY also in nonperpendicular orientations and even to rotate the orientation during flight. Taking advantage of the HYMSY field of view $\left( \pm 22^{\circ}\right)$ and flexibility in rotation angle allowed us to sample anisotropy of reflectance factors of the target area in varying view directions. By installing HYMSY tilted under the UAV so that one extreme of the scan line was viewing nadir, we were able to extend sampling of anisotropy for view geometries up to $44^{\circ}$ zenith angle.

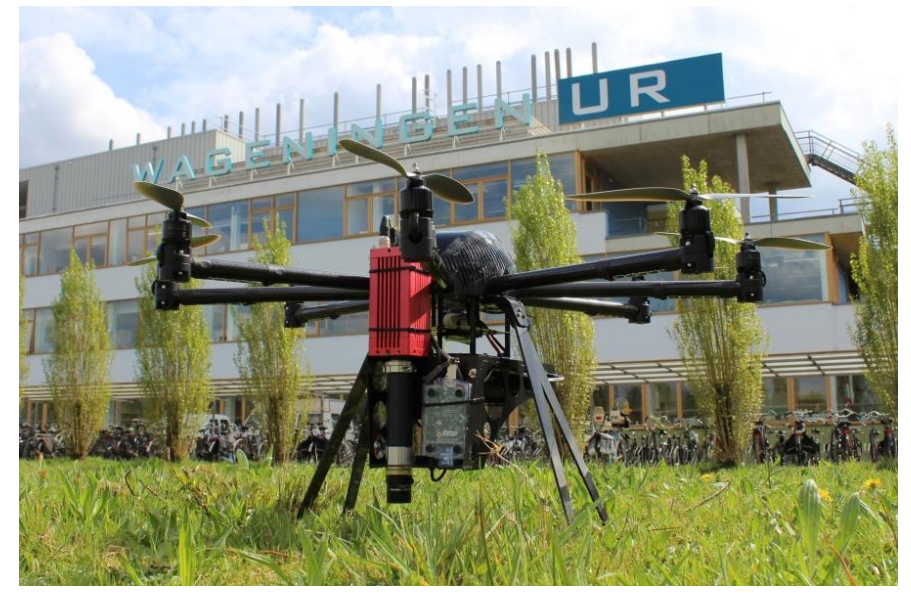

Figure 1. The WageningenUR HYMSY mounted under an Altura AT8 octocopter UAV

\section{LABORATORY HARDWARE}

As a ground reference for UAV based data we have been using the Wageningen University Laboratory Goniometer System (Roosjen, 2012) measurements (Figure 2). The goniometer consists of an ASD FieldSpec 3 spectrometer (350-2500 nm, FWHM 3.5-7.0 nm) mounted on a robotic arm. The robotic arm rotates the spectrometer around the illuminated target and samples the BRF from a series of predefined view directions.

Corresponding author (juha.suomalainen@wur.nl) 


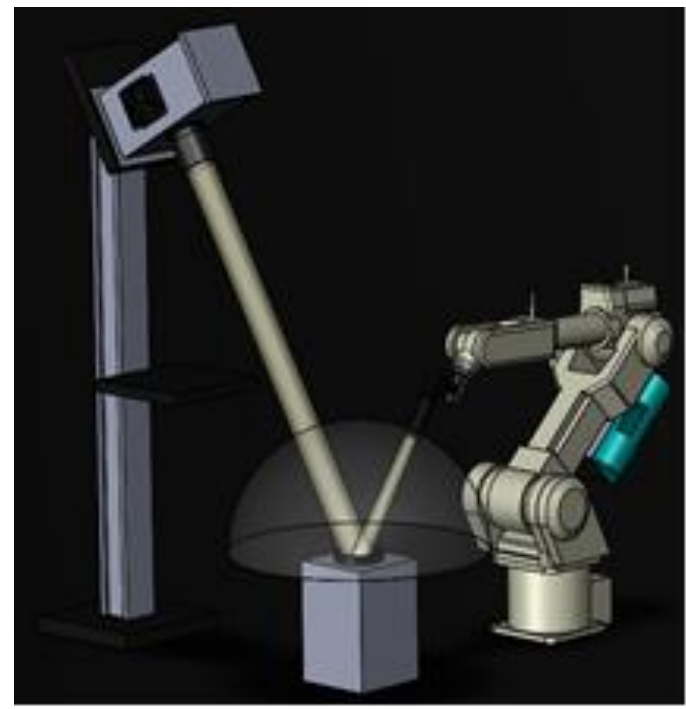

Figure 2. The Laboratory Goniometer System

\section{EXPERIMENTS}

During summer 2014 we performed the first preliminary reflectance anisotropy acquisition tests over a sugar beet field near Wageningen. The HYMSY system was flown over the field in four flight lines where the scan line was aligned parallel to the solar principal plane (figure 3). Each flight line was 80 meters long. The solar zenith angle was $30^{\circ}$ and sky was cloudless during the flight. After the flight, some of the sugar beet plants were taken to the laboratory and measured using the goniometer system. The figures 2 middle and 2 right show the anisotropy of reflectance in the illumination principal plane.

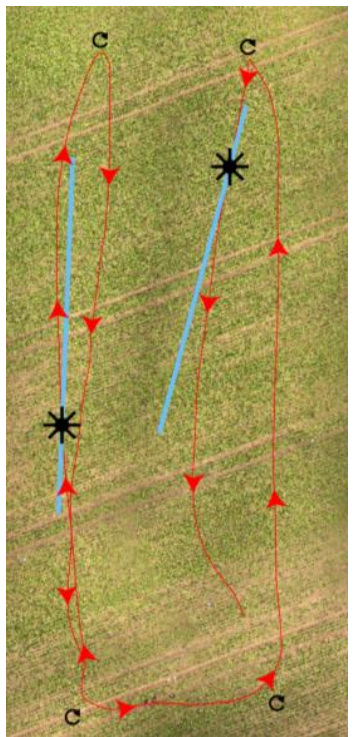

Figure 3. UAV flight path over the sugar beet field in the 2014 experiment.

During spring and summer 2015 a more extensive measurement campaign on vegetation and soil targets was performed. The reflectance factor anisotropies of wheat, barley and potato fields were measured on three days over the growth of the crops. On each day the anisotropy measurement was repeated 4-5 times with different solar zenith angles ranging from $60^{\circ}$ to $40^{\circ}$. In these experiments the UAV was continuously rotated allowing anisotropy measurement, not only in the principal plane but in all diretions with view zenith angle smaller than $22^{\circ}-28^{\circ}$. The first results of these new measurements will be presented in this conference.

\section{RESULTS AND DISCUSSION}

In the 2014 data, both the UAV and goniometer data show a similar trend in reflectance anisotropy, but in goniometer data its amplitude is higher. (figure 4) This is due to the fact that both sample and illumination were slightly different between the measurements. In field the target consisted of a mixture of sugar beet plants and soil, while in laboratory only the single sugar beet plants were measured. The illumination in field was a typical blue sky irradiance with a significant diffuse component especially in short wavelengths. In the laboratory illumination the absence of the diffuse component from the sky and the surrounding plants enhanced the directional effects.
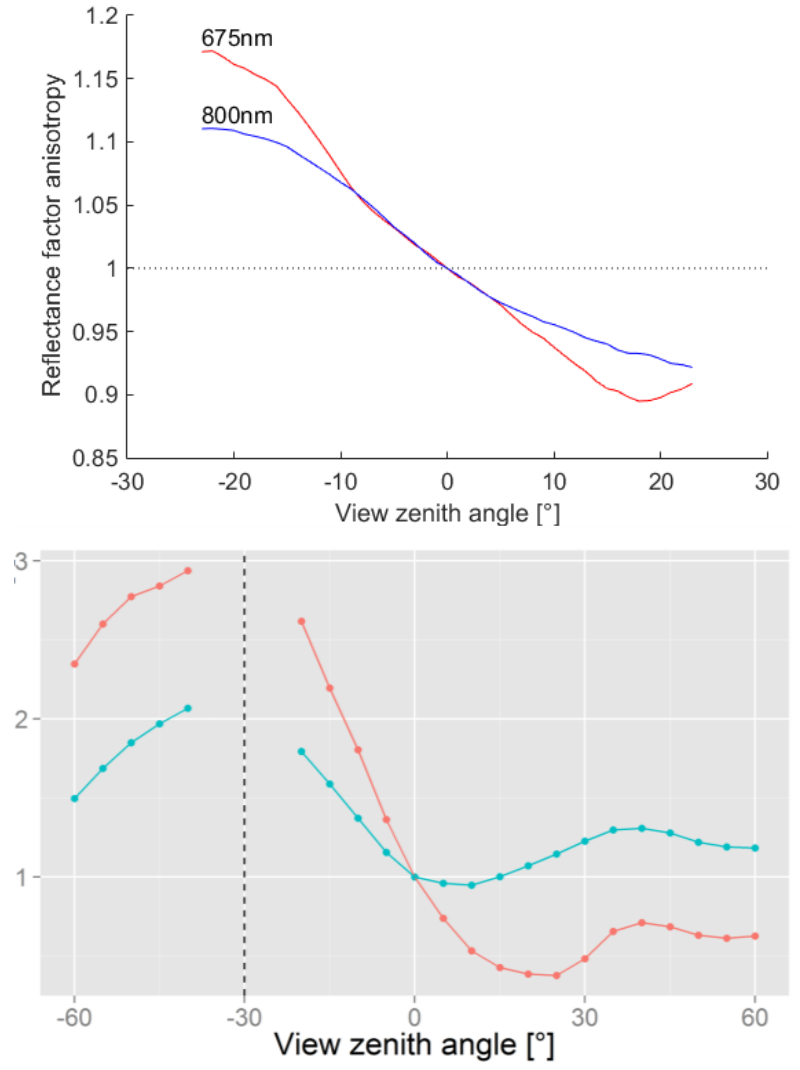

Figure 4. Reflectance anisotropy of sugar beet field in the solar principal plane as measured by the HYMSY (top) and the goniometer (bottom). Sun/lamp zenith angle in both datasets is $30^{\circ}$.

\section{REFERENCES}

Suomalainen, J., Anders, N., Iqbal, S., Roerink, G., Franke, J., Wenting, P., Hünniger, D., Bartholomeus, H., Becker, R. \& Kooistra, L., "A Lightweight Hyperspectral Mapping System and Photogrammetric Processing Chain for Unmanned Aerial Vehicles", Remote Sensing, 2014, 6, 11013-11030. doi:10.3390/rs61111013 
The International Archives of the Photogrammetry, Remote Sensing and Spatial Information Sciences, Volume XL-1/W4, 2015 International Conference on Unmanned Aerial Vehicles in Geomatics, 30 Aug-02 Sep 2015, Toronto, Canada

Roosjen, P.P.J., Clevers, J.G.P.W., Bartholomeus, H.M., Schaepman, M.E., Schaepman-Strub, G., Jalink, H., van der Schoor, R., \& de Jong, A., "A Laboratory Goniometer System for Measuring Reflectance and Emittance Anisotropy", Sensors, 2012, 12, 17358-17371. doi:10.3390/s121217358 